\title{
Gate-Controlled Memristors and their Applications in Neuromorphic Architectures
}

\author{
Eric Herrmann \\ EECS, University of Cincinnati \\ Cincinnati, Ohio \\ herrmaej@mail.uc.edu
}

\author{
Rashmi Jha \\ EECS, University of Cincinnati \\ Cincinnati, Ohio \\ jhari@ucmail.uc.edu
}

\begin{abstract}
We discuss the theory of gated memristive devices, which exhibit continuous states over three orders of magnitude and can be programmed independently of reading. A model is generated by using knowledge of the device physics and fitting the parameters to measured data. The gate-controlled memristor simplifies the implementation of analog artificial neural network architectures significantly. Using this, a very simple architecture is presented, along with a simulation and its performance metrics. The simulated analog neural neural network is able to achieve 88.9 percent accuracy on the MNIST test set. The objective is to demonstrate the advantages that gated memristors can give to analog neural networks.
\end{abstract}

\section{CCS CONCEPTS}

- Hardware $\rightarrow$ Non-volatile memory; Programmable logic elements; • Information systems $\rightarrow$ Storage class memory; • Computer systems organization $\rightarrow$ Neural networks;

\section{KEYWORDS}

Neuromorphic Architecture; Memristor; Circuit Design

\section{ACM Reference Format:}

Eric Herrmann and Rashmi Jha. 2018. Gate-Controlled Memristors and their Applications in Neuromorphic Architectures. In GLSVLSI '18: 2018 Great Lakes Symposium on VLSI, May 23-25, 2018, Chicago, IL, USA. ACM, New York, NY, USA, 6 pages. https://doi.org/10.1145/3194554.3194613

\section{INTRODUCTION}

Optimizing the training and evaluation of artificial neural networks (ANNs) using traditional CMOS architectures has been a topic of research for many years, with varying levels of success[1,3]. The obvious reason for this is the increased interest in applying neural networks to machine learning and artificial intelligence problems, yet the problem is very suited to optimization due to the often parallel nature of the problem. What is not new is the implementation of neural networks as arrays of resistive devices, which had been first proposed in the 1960s[4]. This solution to the problem has long been one of the more interesting research topics into analog computing. The advantages are clear, analog

Permission to make digital or hard copies of all or part of this work for personal or classroom use is granted without fee provided that copies are not made or distributed for profit or commercial advantage and that copies bear this notice and the full citation on the first page. Copyrights for components of this work owned by others than ACM must be honored. Abstracting with credit is permitted. To copy otherwise, or republish, to post on servers or to redistribute to lists, requires prior specific permission and/or a fee. Request permissions from permissions@acm.org.

GLSVLSI '18, May 23-25, 2018, Chicago, IL, USA

(C) 2018 Association for Computing Machinery.

ACM ISBN 978-1-4503-5724-1/18/05 . \$ \$15.00

https://doi.org/10.1145/3194554.3194613 multiplication can be done by simple resistive devices, and sums can be done by combining resistive outputs. It is truly parallel in a dense array. For traditional computing methods, computing the output and weight update of a single layer of a network are both $\mathrm{O}\left(\mathrm{N}^{2}\right)$ operations, but with a resistive crossbar based network, these are constant time operations $(\mathrm{O}(1))$.

Recently, progress has continued to be made on this front. A well-performing architecture using phase change memory (PCM) devices and stochastic bit streams to evaluate and train a deep neural network (DNN) using backpropagation has been proposed [1]. However, this architecture lacks the simplicity many may expect from an analog neural network implementation. What we will discuss in this paper is the implications of a three-terminal (3T) non-volatile memory (NVM) cell on ANNs, and propose a simple architecture for $\mathrm{O}(1)$ evaluation and learning.

The 3T NVM cell behaves as a resistor with a gate to control its resistance. This allows for greatly simplified architectures compared to two-terminal NVM cells, since with 3T devices the read and write lines are seperated. This means CMOS logic can be used at each cell to program it, and all sneak path problems are eliminated when configuring. The device described has continuous programmable states, making it optimal for this problem. [2]

\section{GATED MEMRISTOR}

The gated memristor described and simulated below has been fabricated by our group previously [2]. Here we will discuss the theory and testing results of the device, with a focus on how this information can be applied to device modeling.

\subsection{Theory}

A cross-section of the fabricated device can be seen in Fig. 1. The switching metal oxide used is $\mathrm{SrTiO}_{3}$ (STO), which has been studied for its mobile oxygen ions $\left(\mathrm{O}^{2-}\right)$ and varying electrical properties at different oxidation states. As $\mathrm{O}^{2-}$ migrates, it forms oxygen vacancies $\left(\mathrm{V}_{O}^{2+}\right)$ in the material, in this study we are assuming regions depleted of oxygen exhibit higher conductivity. The accepted transport mechanisms through the material generally support this, with oxygen vacancies promoting n-type ohmic conduction [5] and trapassisted tunneling as well as contact effects. The conduction region of the device is the region between the top electrode (TE) and bottom electrode (BE), a positive voltage on the gate will accumulate $\mathrm{V}_{O}^{2+}$ in this region, and increase conductivity. A negative voltage will deplete this region of $\mathrm{V}_{O}^{2+}$, and increase resistivity.

The fabricated device exhibits continuous states over 3 orders of magnitude. The set and reset powers are measured in $\mathrm{pW}$ for this device[2]. The states are related to the integrated electric field through the STO over time, as will be discussed below. 


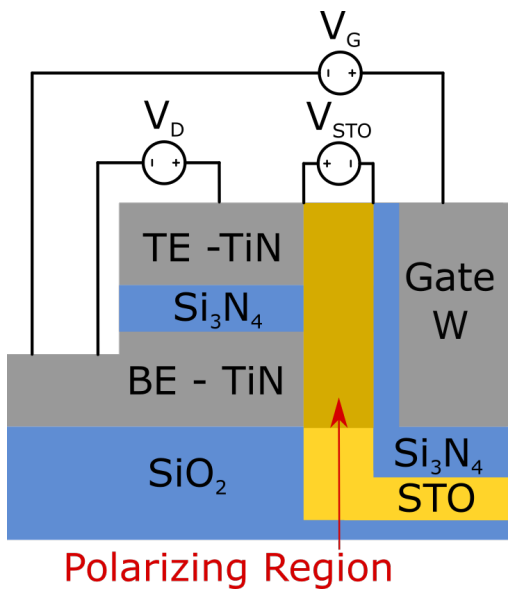

Figure 1: The designed device cross-section. The switching oxide is STO, and the polarizing STO region is between the two electrodes and the gate electrode, where all conduction occurs between the top and bottom electrodes. $V_{S T O}$ is defined as the polarization state of the dielectic (potential drop across it when $V_{G}=0 \mathrm{~V}$ due to the gradient of $\left.V_{O}^{2+}\right) . V_{G}$ is the gate voltage and $V_{D}$ is the read voltage with the $\mathrm{BE}$ as the reference ground.

\subsection{Modeling}

For the purpose of modeling this device, the STO layer is treated as a slowly-polarizing ionic dielectric. That is:

$$
\frac{\partial V_{S T O}}{\partial t}=\frac{V_{a p p}-V_{S T O}}{\tau}
$$

where $\mathrm{V}_{S T O}$ is the internal voltage across the STO as shown in Fig. 1, and $\mathrm{V}_{a p p}$ is the applied voltage. This is saying that the material will exhibit ionic polarization linearly with the applied field (as per the charged particle drift equation). As it polarizes, an internal field opposing the applied field will form (as in all dielectrics). However, due to the low ion mobility in STO[5], this will take a significant amount of time. At steady-state, the STO will be fully polarized to the applied voltage, and so the entire voltage drop applied across the device will be seen across the gate insulating layer $\left(\mathrm{Si}_{3} \mathrm{~N}_{4}\right)$. The parameter $\tau$ is an indication of the ionic mobility and device dimensions, and will be a function of temperature. In our study, it is a fitted parameter and assumed constant. $V_{a p p}$ is defined based on the device structure. In the device shown in Fig. 1, the applied voltage is a function of the gate voltage and the voltage on the TE and $\mathrm{BE}$ :

$$
V_{a p p}=V_{G}-\frac{V_{T E}+V_{B E}}{2}
$$

It is important to discuss the assumptions in this relationship. Firstly, if there is a bias across the TE and BE, there will be more complex fields through the STO than can be described by a single potential drop. Second, the non-ionic polarization in STO would also need to be accounted for, since this reduces the effective field through the STO. In (1), the dielectric constant is assumed to be 0 when it is not polarized (all voltage drop is across the STO, none across the gate insulator), and infinity when it is. Third, the only

\begin{tabular}{c||c|c|c|c|c} 
& $\tau(s)$ & $\mathrm{V}_{T}(V)$ & $\alpha(V)$ & $\beta$ & $\mathrm{g}_{\min }(\log (S))$ \\
\hline \hline Mean & 16.6 & .1739 & .314 & 3.12 & -11.84 \\
\hline STD & .765 & .0571 & .0233 & .2283 & .1949
\end{tabular}

Table 1: The means and standard deviations of the model parameters were extracted from $5100 \mu \mathrm{m}$ devices

particle effect is assumed to be drift, while diffusion likely also plays a part. These assumptions could be accounted for in future work, but as will be seen below, these assumptions do not significantly compromise the model's effectiveness.

Relating this polarization state to the device conductivity is less intuitive than the polarization model. Fitting (1) to the measured data, the $\tau$ can be extracted using voltage sweep data. Since the conductivity of the device should be the same under a given polarization condition, the time constant $(\tau)$ can be determined by increasing and then decreasing the polarization state, and aligning each path. This method is visually explained in Fig. 2(a). The device at DC shows a n-channel MOSFET-like characteristic, with an exponential relationship to polarization state in the subthreshold region, and linear transconductance after the threshold. However, in this voltage region, it was found that the $10^{\text {sigmoid }}$ relationship was more appropriate due to the high leakage current. The current model is shown here:

$$
I_{D}=10^{\beta \operatorname{sig}\left(\frac{V_{S T O}-V_{T}}{\alpha}\right)+\log _{10}\left(g_{\text {min }}\right)}
$$

Where sig is the sigmoid function $\left(\operatorname{sig}(x)=\frac{1}{1+e^{-x}}\right)$, and the fitted parameters are the minimum conductivity $\left(g_{\text {min }}\right)$, the threshold voltage $\left(V_{T}\right)$, and scaling factors $\alpha$ and $\beta$.

These models were fitted to five measured device $\mathrm{V}_{G}-\mathrm{I}_{D}$ sweeps[2] The voltage between the top electrode (TE) and bottom electrode (BE), $V_{D}$, was held at $1 \mathrm{~V}$. The gate voltage $\left(V_{G}\right)$ was swept from $-5 \mathrm{~V}$ to $5 \mathrm{~V}$ and back over the course of 60 seconds, and the current was measured between the TE and BE. Fitting via parameter optimization was done using least squares optimization. The fitted parameters are shown in Table ??, with means and standard deviations on this small set of devices. The results of this model can be seen in Fig. 2.

One major assumption is made in the application of this device. That is that the gate will have high enough capacitance and low enough leakage to retain the device state. This is not an unreasonable assumption, since STO is a high-K dielectric. Realistically, opening the gate terminal would slow the decay rate of the device significantly. However in this study we assume there is no decay due to the trapped charge on the gate.

\section{MEMRISTOR NETWORK}

The non-volatility of such a device makes it useful for applications traditionally handled by memristors. Specifically, here we explore the applications of such a device to evaluation and training of an ANN applied to a classification problem. The third terminal allows for a feedback training signal. This greatly simplifies the control logic required to train this network.

The basic computations in a network involve computing the dot product of the input and the weight matrix, evaluating the activation function, and finally doing training via gradient descent. 


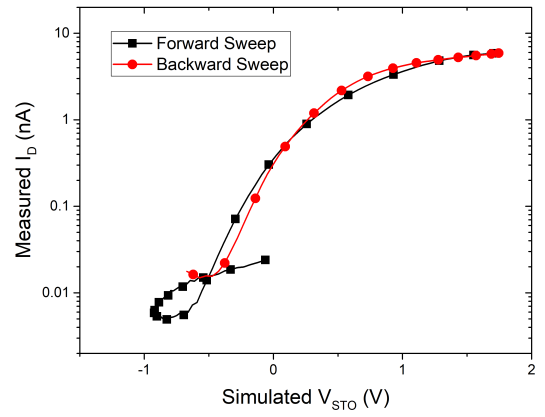

(a)

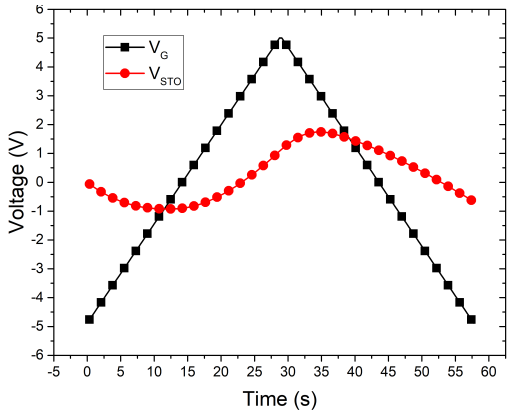

(b)

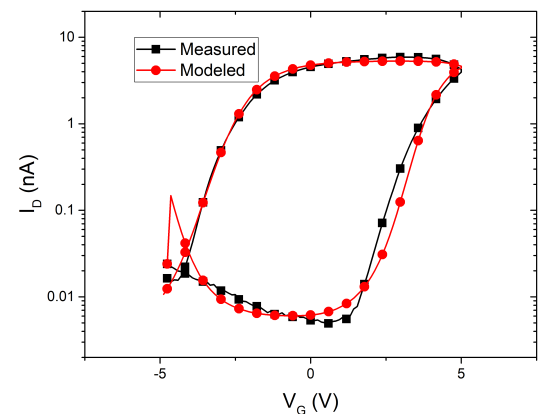

(c)

Figure 2: The model fitness. (a) Measured read current $\left(I_{D}\right)$ at $V_{D}=1 \mathrm{~V}$ against $V_{S T O}$ (simulated polarization state). The curve follows itself in both directions, meaning the time constant is correct, since $I_{D}$ is a function of only $V_{S T O}$ (and $V_{D}$, which is constant). (b) $V_{G}$ against $V_{S T O}$. The simulated dielectic potential ( $\left.V_{S T O}\right)$ lags behind the applied voltage due to low ionic mobility, modeled by (1). (c) The measured and modeled read currents $\left(I_{D}\right)$ in a $V_{G}$ sweep from $-5 \mathrm{~V}$ to $5 \mathrm{~V}$ and back, showing the accuracy of the model and the low drift from the measured value over time (60 seconds).

The dot product can be expanded into a sum of the form:

$$
s_{i}=\sum_{j=0}^{\operatorname{len}(\bar{x})} x_{j} w_{i j}
$$

Where $w$ is the weight matrix, $\bar{x}$ is the input vector, and $\bar{s}$ is the dot product of the two. This can fairly easily be translated to a resistive crossbar array, with the weights equal to the conductivities of the resistors, and $s$ and $x$ being output and input voltages respectively. Using the current law:

$$
0=\sum_{j=0}^{\operatorname{len}(\bar{x})}\left(x_{j}-s_{i}\right) w_{i j}
$$

For all $i$. This can be simplified to:

$$
s_{i}=\frac{\sum_{j=0}^{\operatorname{len}(\bar{x})} x_{j} w_{i j}}{\sum_{j=0}^{\operatorname{len}(\bar{x})} w_{i j}}
$$

Which is the simple normalized weighted sum of the inputs, and has the same effect as (4).

For activation, the easiest activation function in this context would be the heaviside function, which can be approximated with a voltage comparator. However, activation functions are often forgone in output layers, which we will do in this case. Instead, a winner-take-all (WTA) method is used using a multiple input/output dynamic comparator, which returns a 1 for the highest input, and 0 for the rest. This is similar but not equivalent to the softmax function in software based networks, that are commonly used There are many ways to implement such a circuit.

For training, the gradient descent rule is approximated using boolean logic. The traditional gradient descent rule for a single layer is:

$$
\Delta w_{i j}=\eta f_{i}^{\prime}\left(s_{i}\right)\left(y_{i}-\hat{y}_{i}\right) x_{j}
$$

Where $f^{\prime}$ is the derivative of the activation function with respect to $s, y$ is the desired output of the activation function, $\hat{y}$ is the evaluated output of the activation function $(\hat{y}=f(s))$, and $\eta$ is the learning rate.

For our approximation, the derivative of the activation function is removed. Instead, the weights are updated whenever the wrong classification wins, and the derivative is assumed constant. Since all training rules and outputs of the network are binary, the subtraction can be done with boolean logic. There are three possible states for feedback to each dendrite: increase output, decrease output, and do nothing. These are expressed as a 2-bit signal for each output of the network. The first bit of this signal is an output enable (OE), if it is 1 then the weight is updated. Otherwise, the memristor's gate terminal is left open, and no update is applied, so the feedback signal is ignored. The second is the feedback signal, which will be a 1 if the network output should be increased, a 0 otherwise. This is equivalent to subtracting $\hat{y}_{i}$ and $y$, expressed at 3 different logic levels $(-1,0,1)$. The boolean logic for this is simple:

$$
\begin{gathered}
O E_{i}=y_{i} \oplus \hat{y}_{i} \\
\text { feedback }_{i}=y_{i}
\end{gathered}
$$

The OE signal can also be set to 0 to disable all training, which is done in Fig. 3(d).

The second part of updating needs to be done at each device due to the input dependence as shown in (7). Intuitively, if feedback is 0 , then all devices with low input will be potentiated, and all devices with a high input will be depressed, and vice versa if feedback is 1 . The output of this is then gated by the OE signal. This is equivalent to multiplying $y_{i}-\hat{y}_{i}$ and $x_{j}$, if possible values are $(-1,0,1)$. Then the boolean logic for each device is:

$$
V_{\text {update }, i j}= \begin{cases}\overline{\text { feedback }_{i} \oplus x_{j}}, & \text { if } O E=1 \\ \text { open, } & \text { if } O E=0\end{cases}
$$

The actual weight update is simulated using the device model described in (1), (2), and (3). If the update is 'open', the gate is disconnected and so the applied voltage is equivalent to the potential 


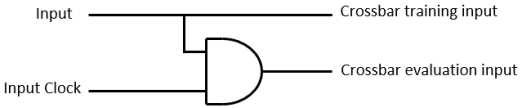

(a)

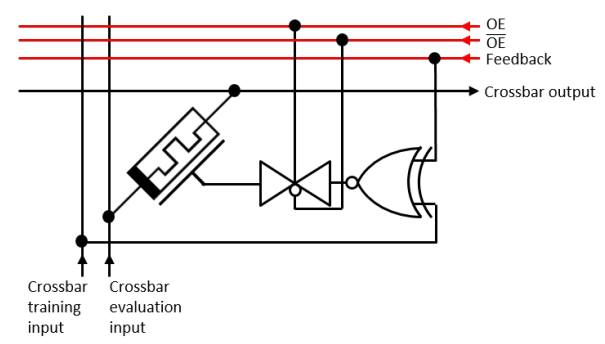

(b)

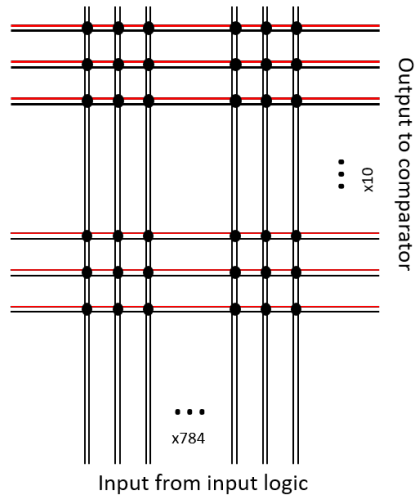

(c)

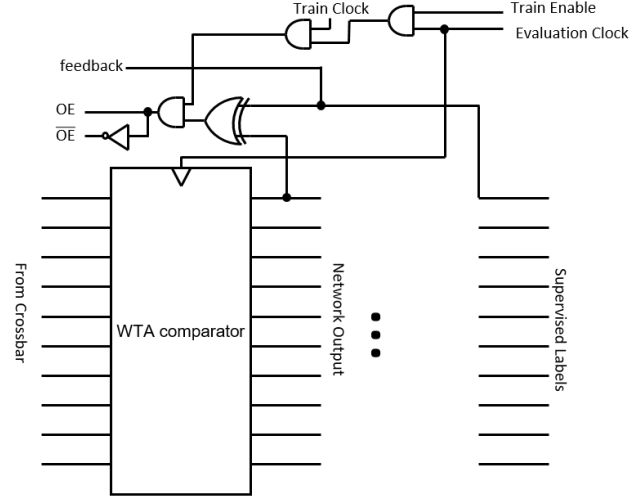

(d)

Figure 3: The network architecture. (a) The input logic. This will enable the input while evaluating the network, but once the comparator evaluates, the inputs will disable. This is repeated for each of the 784 inputs. (b) The crossbar intersection circuitry. There is a single gated memristor, a transmission gate, and an xnor at each intersection. (c) The crossbar array, there is a 784-bit binary input, and 10 analog outputs. Each intersection contains a memristive device, and inputs come from the input circuitry. Feedback signals (OE and $f e e d b a c k$ ) from the output are marked with red. (d) The output contains a 10-input winner-take-all comparator, which compares the outputs of the crossbar. A one-hot label vector is provided to this block when training. There is some simple control logic that operates on each output/label pair. Training can be disabled with a one bit signal.

stored in the dielectric. (2) can be rewritten with the updated terminology:

$$
V_{a p p, i j}= \begin{cases}V_{u p d a t e, i j}-\frac{V_{x_{i}}+V_{s_{j}}}{2}, & \text { if } O E=1 \\ V_{S T O, i j}, & \text { if } O E=0\end{cases}
$$

The final term in (4) is the learning rate, $\eta$. This is proportional to the time the signal is applied to the gate, and is the same for all devices.

This is the entire network definition, the implementation can be seen in Fig. 3. Realistically, this network would be fabricated with all digital circuitry in silicon, and then the memristive devices would be added in the metal layers (back end of line, BEOL).

The major approximation made with this implementation is that all signals are binary except for the network output, which is then binarized with a WTA comparator. The inputs also need to be binary to allow for them to be used in the weight update. Additionally, the error function is binary, which could cause problems with reaching a good error minimum. However, when not training, analog inputs can be used to evaluate the network.

For usage, the input vector is displayed, and the evaluation/training is controlled with the evaluation clock. It was found that the performance could be improved by grounding the TE/BE terminals of the memristive device when training, this allows the training to be more uniform across devices, this is not strictly necessary however. So, it is assumed that the input vector is grounded after evaluation. This could be accomplished using the inverted evaluation clock through a short delay to gate the input vector, as shown in Fig. 3(a). The training period is then allowed for a set amount of time, while the evaluation clock is high. The learning rate can be modulated by increasing or decreasing this clock frequency/duty

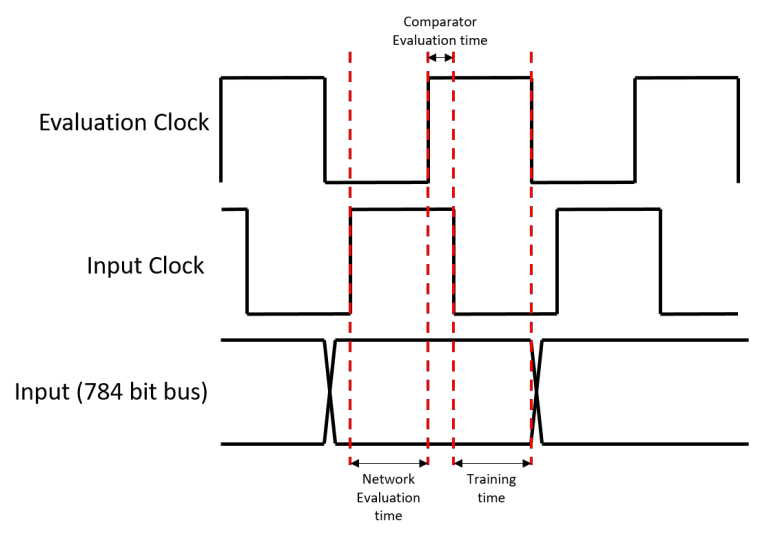

Figure 4: The timing diagram during training, including the evaluation clock which controls the comparator evaluation, the input clock which controls the crossbar evaluation, and the input change times. The network evaluation period is the time the network is allowed to settle, the required time will be a function of the network parasitics. The comparator evaluation time is the time allowed for the comparator to evaluate the crossbar output before removing the input. After the input is removed, the weights are updated for a set amount of time, $100 \mu \mathrm{s}$ in this experiment.

cycle. The network described above was constructed and simulated using Python. Discrete time steps were used with a $d t$ of $100 \mu \mathrm{s}$. The traditional MNIST handwritten number dataset was used for testing, with 784 inputs and 10 outputs. For training, as discussed 


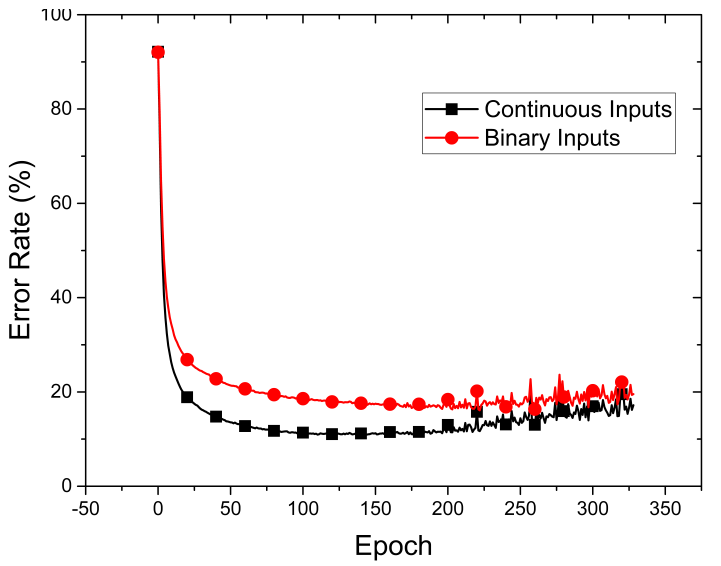

Figure 5: Network error rate on the 10,000 image MNIST test set over 330 epochs. The binary input vector representation rounds the MNIST pixel values to high or low, and is represented by the two logic levels. The continuous input vector scales the input voltage between the two logic levels based on the grayscale pixel value. The The minimum error rate is $\mathbf{1 0 . 8 4}$ percent with continuous coding, and $\mathbf{1 6 . 0 9}$ percent with binary.

above, the grayscale inputs were rounded to 0 or 255 to create a binary representation (and represented as $0 \mathrm{~V}$ or $V_{D}$ internally). At each timestep, a training vector was presented. For the simulation, the network was assumed to evaluate instantaneously. The memristive weights are then trained for a full $100 \mu$ s on each sample, with the input vector grounded as discussed. Since the weights are trained for the full $d t$, this can be thought of as the learning rate, the longer the training signal is applied, the more the device will depress/potentiate. This timing of input and update cycles can be seen in Fig. 4. Realistically, the evaluation clock duty cycle, input clock phase shift, and frequency would be tuned to optimize training speed, learning rate, and performance.

After each set of 60,000 training samples, the network is evaluated by disabling training and testing it using the 10,000 sample test set. This would physically be done by disabling the 'Train enable' signal, and then directly observing the WTA comparator's one-hot output.

The memristive device model used is described above, with the model parameters initialized to the means of parameters shown in Table ??. Logic levels of $0 \mathrm{~V}$ and $2 \mathrm{~V}$ were used for the simulation, which is significant because it affects the operating region of the gated memristors. Initial device states were assumed to be normally distributed around their ground states $\left(V_{S T O}=0\right)$ with a standard deviation of $.1 \mathrm{~V}$.

\section{RESULTS}

The network was evaluated over 330 training epochs. The network was trained using the binary vector as discussed above, but can be evaluated using continuous values (analog input is the grayscale pixel values scaled between the two logic levels), which increases

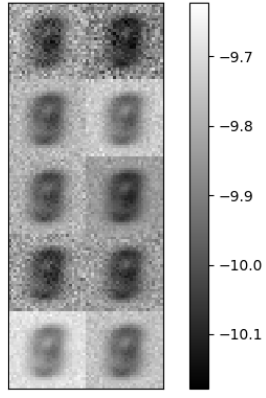

(a)

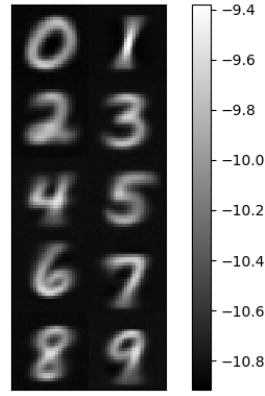

(b)
Figure 6: Conductance matrices after training. The images are arranged into a $2 X 5$ array corresponding to the 10 network outputs. For each, there is a $28 \mathrm{X} 28$ array which corresponds to the $\mathbf{7 8 4}$ gated memristor devices. The color scale is in terms of $\log _{10}(w)$, where $w$ is the conductance of the gated memristor. (a) The conductance matrix using the WTA comparator described in this paper. (b) The conductance matrix trained using 10 normal output comparators instead, with a threshold voltage of $\frac{V_{D}}{2}$.

performance. This could be accomplished by digital to analog converters (DACs) on the inputs. The network performance on the 10,000 item test set over the 330 epochs is shown in Fig. 5.

Using a binary input vector, as in training, results in a penalty of about 5.5 percent accuracy compared to evaluating the network using continuous values. The error rate converges to over 89 percent accuracy with a continuously coded input, but then begins to degrade as the devices begin to become saturated. However, the network converges to a good minimum before running out of conductance range. Avoiding the saturation of devices would need to be addressed for physical realization.

Fig. 6(a) shows the conductances of the gated memristors in the trained array. There is little visual indication that the network has been trained, which is a result of the WTA comparator, which only enforces separation between outputs, but has no control over the global output level. Fig. 6(b) shows the conductances trained with a similar network to the one described in this paper, except with two-input comparators at the output (heaviside activation) instead of the WTA comparators for training. This hurts accuracy, since multiple outputs can win. Even with a WTA comparator to evaluate the network after training, the performance is worse. The purpose of showing this image is to demonstrate the effect of training on the network conductances. Further work can explore this architecture, these networks may be more noise resistant and could prevent weight saturation.

\section{DISCUSSION}

The merits of this network are not necessarily due to its performance, indeed many others have shown better performance with 
single layer neural networks. However, this network is very simple and has very little peripheral circuitry, made possible by the use of 3T memristors. This is a binary voltage level system, which makes implementation straightforward with CMOS technology. Many similar implementations use spiking networks or some kind of pulse width modulation (PWM) to describe continuous values, with a capacitor for integration. In this case, the analog signals are evaluated directly without the need for integration, which can potentially speed up evaluation time. However, the price is that the gradient descent algorithm can only be approximated and the system is less resistant to noise. Even so, for simple problems this may be sufficient.

There are many variations to this. Adding noise will cripple system performance, due to the the similarity of network outputs. This is because once the network obtains the correct output, it will not differentiate the outputs more, due to the binarization of the outputs. This effect could be mitigated by adding some capacitance to the comparator input and leave some integration time. The differentiation could be improved by adding small gated static resistors to ground on the WTA comparator inputs. By only connecting the resistor corresponding to the true value, the output will be lowered during training. This will increase differentiation, and help with noise resilience. Or, a stochastic approach could be taken, by intentionally adding additional noise during training to help differentiate the signals more. As discussed above, a different activation method could also be used, where the outputs are not directly competing during training. These experiments will be left for future work.

There are some obvious improvements that could be made, and will be examined in future work. The physical training time would be very long due to the the low ionic mobility in STO, this could be improved in several ways as has been previously discussed[2]. Adding layers could improve the accuracy. Backpropogation could be implemented in a similar fashion, since all outputs are binary they could be stored in registers and the network could be evaluated in reverse.

\section{CONCLUSION}

The implications of a gated memristor in neurmorphic applications could potentially be disruptive. It allows for very simple architectures such as the one described here. This is because there doesn't need to be complex logic to control evaluation and training cycles, instead both evaluation of the network and training can be done with no need to share read/write terminals. This also means potential sneak path problems with configuring devices is eliminated. Additionally, the power consumption of such a device is far less than a similar 2-terminal device [2].

More complicated architectures could be developed as well. For instance, more accurate analog operations could be done, as far as analog subtraction and signal mixing, to more adequately approximate the gradient descent algorithm. Or, digital logic could be added, as demonstrated[1], to do stochastic multiplication using an AND gate at the crossbar intersection. Activation and their gradient calculations could be done in digital logic, and then utilize the parallel nature of the crossbar array to do weight updates.

Future work will include varying the device fabrication process to improve characteristics, as well as additional measurements to get a more accurate parameter distribution. The learning architecture can be modeled with distributed device parameters and with added noise. Noise resilience measures could also be tested for effectiveness. The architecture could be extended to multiple layers; Since all signals are binary, the feedback signals can easily be stored in registers to enable backpropagation or even recurrence.

\section{ACKNOWLEDGMENTS}

The work was supported by the National Science Foundation under Award ECCS 1556294.

\section{REFERENCES}

[1] Tayfun Gokmen and Yurii Vlasov. 2016. Acceleration of Deep Neural Network Training with Resistive Cross-Point Devices: Design Considerations. Frontiers in Neuroscience 10 (2016), 333. https://doi.org/10.3389/fnins.2016.00333

[2] Eric Herrmann, Andrew Rush, Tony Bailey, and Rashmi Jha. 2018. Gate Controlled 3-Terminal Metal Oxide Memristor. IEEE Electron Device Letters (Feb. 2018). https: //doi.org/10.1109/LED.2018.2806188

[3] Cory E. Merkel, Nakul Nagpal, Sindhura Mandalapu, and Dhireesha Kudithipudi. 2011. Reconfigurable N-level memristor memory design. In The 2011 International Joint Conference on Neural Networks. IEEE. https://doi.org/10.1109/ijcnn.2011. 6033622

[4] Karl Steinbuch. 1961. Die Lernmatrix. Kybernetic 1 (1961), 36-45.

[5] J.J. Wang, H Huang, Thorsten Bayer, Ali Moballegh, Ye Cao, Andreas Klein, Elizabeth Dickey, Douglas L. Irving, Clive Randall, and Long-Qing Chen. 2016. Defect chemistry and resistance degradation in Fe-doped $\mathrm{SrTiO} 3$ single crystal. Acta Materialia 108 (04 2016), 229-240. 